Rev. Psicol. (Arequipa. Univ. Catól. San Pablo) / Año 2020 / Vol 10 / Nº 2 / pp. 57-75

ISSN 2306-0565 versión impresa / ISSN 2311-7397 versión on line

(9) $(\Theta \Theta \Theta$

Esta obra está bajo una Licencia Creative Commons

Atribución 4.o Internacional (BY-NC-ND)

\title{
SATISFACCIÓN LABORAL Y VARIABLES SOCIODEMOGRÁFICAS EN LOS TRABAJADORES DE UNA EMPRESA FERROVIARIA EN LA CIUDAD DE AREQUIPA
}

\author{
JOB SATISFACTION AND SOCIODEMOGRAPHIC VARIABLES \\ OF WORKERS FROM A RAILWAY COMPANY IN THE CITY OF AREQUIPA
}

\author{
María Fernanda Chocano Córdova y Cristina Isabel Pacheco Paredes \\ Universidad Católica San Pablo, Arequipa, Perú
}

\begin{abstract}
Resumen
El objetivo de este estudio es determinar las diferencias que existen en la satisfacción laboral de los trabajadores de una empresa ferroviaria en función de las variables sociodemográficas. Los participantes de la investigación fueron 83 trabajadores de una empresa ferroviaria, los cuales fueron evaluados en una clínica de la ciudad de Arequipa, donde se les aplicó una entrevista y la Escala de Satisfacción Laboral de Sonia Palma (SL-SPC), que se compone de siete factores. Las variables sociodemográficas que se tomaron en cuenta fueron la edad, el área de trabajo y el cargo. Se encontraron diferencias significativas en la satisfacción laboral, en función del área de trabajo y el cargo, mas no en función de la edad.
\end{abstract}

Palabras clave: Satisfacción laboral, edad, área de trabajo, cargo, psicología organizacional.

\begin{abstract}
The objective of this study was to determine the differences that exist in the job satisfaction of railway company workers based on sociodemographic variables. The research participants were 83 workers from a railway company who were evaluated in a clinic in the city of Arequipa. An interview and the Sonia Palma Job Satisfaction Scale (SL-SPC), composed of seven factors, were administered. The sociodemographic variables that were taken into account were age, work area,
\end{abstract}


and position. Significant differences were found in job satisfaction, depending on the work area and the position, but not depending on age.

Key words: Job satisfaction, age, work area, job title, organizational psychology.

\section{Introducción}

Las investigaciones acerca de la satisfacción laboral se remontan a autores como Taylor, quien en 1911 se pronunció acerca del tema y es también quien sugiere algunas pautas para generar una mayor eficacia y aumentar la productividad en los trabajadores (Schultz, 1991). En ese sentido, la satisfacción laboral es uno de los factores más relevantes dentro del ámbito organizacional, ya que se encuentra estrechamente relacionada a la productividad laboral de las personas, ya que una persona con un nivel de satisfacción laboral óptimo, es más competente que una persona cuya satisfacción laboral no es buena.

Además, según Alonso (2008), cuando las personas se sienten poco satisfechas en su centro de trabajo, el porcentaje de ausentismo y renuncias suele ser elevado, y pueden darse diversos trastornos tales como ansiedad y depresión, que se encuentran estrechamente relacionados a esta insatisfacción del trabajador. Por el contrario, los trabajadores satisfechos, se muestran más saludables y motivados para desenvolverse en sus tareas diarias.

Diversas investigaciones revelan que en el Perú, existe un gran porcentaje de insatisfacción laboral, es por ello que los investigadores se interesan cada vez más; por el estudio de este tema, dentro de los que resaltan los estudios de León (1981; León, \& Sepúlveda, 1979), por los pioneros en el campo, o los de Palma (1999), quien desarrolló una escala para medir la satisfacción laboral, que ha sido empleada en diversas investigaciones. En la ciudad de Arequipa, las investigaciones de satisfacción laboral, se han centrado en las relaciones entre la satisfacción laboral y la felicidad (Gamero, 2013) o sobre su asociación con el salario, las relaciones interpersonales, la promoción en el empleo, la organización del trabajo y el reconocimiento del trabajador (Arias, \& Justo, 2013).

Así, el estudio es relevante porque permite generar nuevos conocimientos sobre el campo de la satisfacción laboral en un contexto que no ha sido previamente estudiado, y en relación a variables sociodemográficas tales como edad, área y cargo, que podrían implicar ciertas diferencias en la conducta de los trabajadores. En tal sentido, la presenteinvestigación pretenderesponder a la siguientepregunta deinvestigación: ¿Qué diferencias existen en la satisfacción laboral de los trabajadores de la empresa ferroviaria en función de variables sociodemográficas tales como edad, área de trabajo y cargo?

\section{Modelos teóricos sobre la satisfacción laboral}

Una de las primeras definiciones de la satisfacción laboral, fue dada por Hoppock 
(1935), quien consideraba importantes ciertas variables que van a definir la satisfacción de la persona dentro del trabajo: una de ellas es la satisfacción propiamente dicha y el trabajo que realiza la persona, y que forman parte de la satisfacción global con la vida y se relacionan con las capacidades que tiene la persona para adaptarse a diversas situaciones y comunicarse con los demás. También se encuentran otras variables como lo son el nivel socioeconómico y la propia preparación que tiene la persona para el trabajo.

Otro aporte fue dado por Herzberg, que afirma que la satisfacción real de la persona hacia su trabajo comprende factores higiénicos y motivacionales, los primeros se relacionan con el ambiente de trabajo y los segundos con las características del trabajador. Por ello, también se les llama factores extrínsecos e intrínsecos, respectivamente. En cuanto a los factores intrínsecos se dice que son aquellos que están relacionados a la raíz de las tareas y son manejados por la misma persona, ya que están dentro de ella y se relaciona con sigo misma, y en cuanto a los extrínsecos son aquellos que encontramos en el ambiente de la persona y dan lugar a las condiciones en las que se desarrolla su trabajo (Herzberg, Mausner, \& Snyderman, 1959).

Para Herzberg (2004) los factores higiénicos los cuales son extrínsecos a la persona, son tomados como negativos en la satisfacción laboral, ya que si existe alguna deficiencia en estos, serían la causa para que una persona se sienta insatisfecha en su centro de trabajo, mientras que los motivacionales son tomados como positivos, ya que son los principales generadores de satisfacción laboral. Pero señala que ambos factores deben ser considerados independientemente uno del otro, ya que si se trabaja sobre los factores higiénicos no es de esperar que la satisfacción laboral aumente y además un menor nivel en los aspectos motivacionales puede afectar al factor de la motivación. Aunque este supuesto ha sido puesto en duda (León, \& Sepúlveda, 1979), la teoría de Herzberg ha dado pie a importantes teorizaciones como el modelo de las características del puesto y el enriquecimiento del trabajo.

Autores como Hackman y Oldham (1976) exponen que la satisfacción laboral se ve determinada muchas veces por las características propias del puesto que ocupa cada persona, por ello si se trabaja sobre estas, es decir, se modifican estas condiciones laborales de manera positiva, se espera que la satisfacción laboral aumente. Los autores concluyen, además, que el trabajo se enriquece y se vuelve más productivo cuando el nivel de control sobre el trabajador disminuye, y en cambio se le otorga más libertad para que pueda actuar de acuerdo a lo que él vea conveniente, respecto a las tareas que desempeña y las decisiones sobre las mismas.

Otro modelo explicativo de la satisfacción laboral, formulado por un importante teórico sobre el tema, es la teoría de la discrepancia (Locke, 1968), que señala que la satisfacción laboral es un estado emocional placentero que se da como resultado de la valoración del trabajo que 
realiza la persona y como un medio de los logros de los valores laborales (Locke, 1976). También se entiende la insatisfacción laboral como un estado emocional que no causa placer en las personas y se da como resultado de una falta de valoración positiva del trabajo que desempeña la persona, y que puede ser percibido como una fuente importante de estrés y frustración (Locke, 1984).

Otro modelo importante sobre la satisfacción laboral, es el dinámico, que tiene una perspectiva interaccionista, y que fue formulado por Bruggemann (1974). Este modelo no solo se interesa por el nivel de satisfacción laboral que existe en la persona, sino sobre todo, por aspectos cualitativos de la misma. Según este autor, es necesario que la persona tenga responsabilidad y poder sobre el trabajo que realiza, pues el sentimiento de control sobre su labor aumenta la satisfacción laboral que éste pueda manifestar.

Por otro lado, Rodríguez (2009), define la satisfacción laboral como la medida en que son cubiertas ciertas necesidades que pueda tener el trabajador y la magnitud con el que éste ve realizadas las diferentes aspiraciones que puede tener en su ambiente laboral, ya sean de tipo social, personal, económico o higiénico. Este enfoque está inspirado en la teoría de la motivación de Maslow (1943), que diferencia entre cinco tipos de necesidades. Este autor comienza por las necesidades fisiológicas, que están relacionadas a la alimentación, el sueño, el sexo, etc.; siguen las necesidades de seguridad, que implican la necesidad que tienen las personas por preservar su seguridad y aluden el instinto de supervivencia. En tercer lugar se encuentran las necesidades sociales que suponen la necesidad de pertenecer a un grupo dentro de la sociedad y la aprobación dentro de éste. Sobre estas necesidades, se ubican las necesidades de autoestima que son producto de nuestras relaciones sociales, y finalmente, como quinto nivel, se tienen las necesidades de autorrealización que devienen de la autovaloración positiva y de un sentido de vida que desarrolla la persona.

Otro autor que planteó una teoría de la satisfacción laboral es Adams (1963), quien formula su teoría de la equidad, dentro de la cual afirma que lo que desencadena la motivación en la persona es la percepción de igualdad en el trabajo, y da como resultado la concordancia entre los factores resultado/aporte hacia la organización. En cuanto a los aportes por parte de la organización cabe mencionar: los beneficios que ésta brinda a la persona, la remuneración, período vacacional, reconocimiento de horas extras, gratificación por la consecución de objetivos planteados y otros que sean aportes directos de la empresa al trabajador. Por otra parte, con respecto a aportes personales, el autor refiere elementos tales como: el tiempo que la persona dedica a su trabajo, el compromiso que genera hacia la organización en sí misma, el trabajo que realiza, la concentración que conlleva realizar su trabajo y el esfuerzo que dedica sobre el mismo.

La teoría de Aldefer (1969) encuentra relacionada en cierta medida con la de 
Maslow, sosteniendo que los trabajadores tienen también una jerarquía de necesidades, pero estas tienen solo tres niveles, directamente relacionados con el entorno laboral. Estos niveles son: existencia, relación y crecimiento; que pueden asociarse a las necesidades fisiológicas, sociales y de autorrealización de Maslow, respectivamente.

En nuestro país, Palma (1999) define a la satisfacción laboral como una tendencia, que es parcialmente estable hacia el trabajo que realiza la persona, y se encuentra fundamentada en ciertos valores y creencias que son perfeccionados según su experiencia ocupacional. $Y$ ha planteado que la satisfacción laboral se relaciona con los siguientes factores:

1. Condiciones físicas y/o materiales: Los elementos ya sean materiales o infraestructura en donde se desenvuelve la labor diaria de trabajo y se constituye como facilitador de la misma.

2. Beneficios laborales y/o remunerativos: Es el grado de complacencia que se tiene en relación con el incentivo económico normal/mensual u otros adicionales como pago por las diversas labores que realiza.

3. Políticas administrativas: Es el grado de acuerdo que se tiene ante los lineamientos o normas de la institución que están dirigidas a regular la relación laboral y asociada directamente con el trabajador.
4. Relaciones sociales: El grado de satisfacción que tiene la persona frente a la relación con los demás miembros de la organización, con quienes tiene en común actividades laborales diarias.

5. Desarrollo personal: Esta es la oportunidad que se le da al trabajador dentro de la empresa, de hacer actividades las cuales son significativas en cuanto a su autorrealización.

6. Desempeño de tareas: Es el valor con el que se relaciona el trabajador con las actividades diarias en la entidad/ empresa en la que labora.

7. Relación con la autoridad: Es el valor que le da el trabajador a la relación con el jefe directo y a las tareas diarias.

\section{Aspectos asociados \\ a la satisfacción laboral}

Varios estudios han asociado la satisfacción laboral con diversas variables sociodemográficas de los trabajadores. Hong y Giannakopoulus (1994), por ejemplo, afirman que la satisfacción laboral se relaciona con la edad y el sexo, de modo que los trabajadores varones de mayor edad son los más satisfechos. Por otro lado Fernández-Ballesteros (1997) indica que son las mujeres y los trabajadores más jóvenes, quienes presentan mayores niveles de satisfacción en el trabajo. Clemente, Molero y González (200o) encontraron que los grupos que tienen las edades entre 41 a 65 años son los menos satisfechos en comparación a los grupos de edad que van entre los 16 a 40 años, ya 
que a partir de los 40 años es cuando se da un declive de la satisfacción que tiene la persona en cuanto a sí misma. En ese sentido, Argyle (1987) afirma que a partir de los 50 años comienza a disminuir la satisfacción con diversos aspectos de la vida de la persona, no solo la satisfacción laboral, ello debido a los procesos de envejecimiento y el declive de algunas capacidades físicas y cognitivas.

Alonso (2008) descubrió que la satisfacción en las mujeres es mayor que la de los hombres en la mayoría de niveles, ya que para ellas son importantes los factores extrínsecos del trabajo, los cuales están relacionados a la comodidad, ya sea el horario, el lugar de trabajo o la distribución del tiempo, lo que les permite desenvolverse en dos ámbitos importantes en sus vidas, como son el laboral y familiar. También reportó una diferencia entre los intereses de los trabajadores en función del sexo, ya que las mujeres le dan más importancia a las relaciones interpersonales, mientras que los hombres se muestran más interesados con el salario que perciben y la posibilidad de desarrollarse.

Si bien los primeros estudios sobre la satisfacción laboral indicaban que el salario era una variable muy importante en la percepción del trabajador sobre su entorno laboral (Borjas, 1979), estudios más recientes indican que los trabajadores con mayor grado de satisfacción, son los que tienen mejores posibilidades de desarrollo personal y profesional. Por ejemplo, Alonso (2006) hace referencia que la satisfacción laboral para los trabajadores se encuentra relacionada con la percepción de un buen pago, además de ser tomados en cuenta para las decisiones de la empresa, con oportunidades de crecimiento, ascenso y posibilidades de aprendizaje; lo que se verá reflejado en el óptimo desempeño de los trabajadores. Por otro lado, aquellos trabajadores que consideran no ser bien remunerados por el trabajo que realizan, y encontrarse expuestos a malos tratos, o realizar tareas invariables sin posibilidad de desarrollo, tienen un menor rendimiento y un nivel más alto de insatisfacción, y por ende improductividad laboral. Por ello, como señalan Carrillo, Martínez, Gómez y Meseguer (2015), la satisfacción laboral es un reflejo de la condición de vida y bienestar de las personas que trabajan en una organización.

Para Fairbrother y Warn (2003) las condiciones de trabajo son elementos primordiales a la hora de desenvolverse de manera eficiente en las actividades laborales, es por ello que el interés en estas condiciones, así como la salud y seguridad es cada vez mayor, ya que todo esto se encuentra relacionado con la productividad y eficacia de la empresa, lo cual repercute en el nivel de satisfacción de los trabajadores, quienes se muestran satisfechos en el momento que se desenvuelven en un ambiente tanto seguro como saludable. En ese sentido, Probst (200o) afirma que cuando el trabajador percibe falta de seguridad en su trabajo, el nivel de satisfacción laboral es menor.

En consecuencia, es importante que dentro de la organización se tenga un buen ambiente para trabajar y que en 
éste, las personas se sientan a gusto, con comodidad y seguridad. Pero esto también incluye los aspectos interpersonales, es decir, las relaciones socio-afectivas en el entorno laboral, entre los compañeros de trabajo, con los superiores y los subordinados (Arias, \& Justo, 2013). Cuando estas relaciones son positivas entre los trabajadores de la organización, ellos se sienten apoyados, cohesionados y perciben que pertenecen a la organización, por tanto las actitudes hacia su trabajo también serán positivas (Chiavenato, 2009).

Otro dato importante por considerar en la satisfacción laboral, es la percepción positiva de la formación de la persona y el reconocimiento que ésta tenga en su trabajo. En ese sentido, los resultados de investigaciones realizadas en el campo sanitario indican que los trabajadores que se siente mejor reconocidos, a través de diferentes medios, son los que exhiben mayores niveles de satisfacción laboral (Pérez-Ciordia, Guillén-Grima, Brugos, \& Aguinaga, 2013).

Finalmente, no puede obviarse que en las últimas décadas, los escenarios laborales han cambiado mucho con las nuevas tecnologías, priorizándose la flexibilidad y el aprendizaje en el trabajo, de modo que los trabajadores que tengan mayor capacidad de adaptación a su trabajo y los que tengan mayor comprensión de las tecnologías de la información y la comunicación, serán los que se sientan más satisfechos (Chiavenato, 2009).

\section{Método}

\section{Diseño de Investigación}

En el presente proyecto se utilizó un diseño de investigación de tipo no experimental, el cual realiza las observaciones necesarias sin necesidad de intervenir o modificar las variables (Sousa, Driessnack \& Mendes, 2007). Asimismo, se trata de una investigación de tipo comparativo porque se pretende analizar las diferencias entre la satisfacción laboral en función de la edad, el área de trabajo y cargo, lo cual remite a la tipología de estudios asociativos según Ato, López y Benavente (2013).

\section{Participantes}

La muestra está conformada por 83 trabajadores de una empresa ferroviaria, que fueron seleccionados de manera no probabilística, por medio de la técnica de muestreo por cuotas. En función a la edad, se presenta un promedio de 36 años con una desviación estándar de \pm 8.17 dentro de un rango de 24 a 62 años de edad. Con referencia a los más jóvenes, estos representan un $32.5 \%$, cuyas edades se encuentran entre 24 y 30 años.

En relación al área de trabajo, el $4.8 \%$ de los trabajadores son administrativos, los cuales ocupan los siguientes cargos: 1.2\% jefes, el 1.2\% asistente de compras, el 1.2\% coordinador de relaciones comunitarias y el $1.2 \%$ administrador de base de datos. En cuanto al área de transportes, estos representan el 38.6\% de la muestra, dentro de los cuales se presentan los siguientes cargos: el $24.1 \%$ jefes de tren, el $12 \%$ 
maquinista, el $1.2 \%$ operador de tracto remolcado y, el $1.2 \%$ jefe de transportes. Finalmente, el 56.6\% de la muestra, está conformada por el área mecánica, dentro de la cual se presentan los siguientes cargos: el $2.4 \%$ ayudante de track mobile, el $10.8 \%$ ayudante de grúa, el $1.2 \%$ operador de tapas y muestreo, el $2.4 \%$ asistente de jefe de patio, el $12 \%$ operador de grúa, el 1.2\% ayudante de almacén, el $2.4 \%$ maestro mecánico, el $8.4 \%$ jefe de patio, el $1.2 \%$ ayudante de mecánica, el 2.4\% maestro especializado, el $1.2 \%$ maestro electricista, el $7.2 \%$ operador de track mobile, el $2.4 \%$ porta señal y el $1.2 \%$ jefe de almacén.

\section{Instrumentos}

Se aplicó la Escala de Satisfacción Laboral (SL-SPC) creado y validado por Sonia Palma (1999) en la ciudad de Lima, y tiene como objetivo evaluar la satisfacción laboral en trabajadores. Esta escala consta de 36 ítems distribuidos en siete factores:

1. Condiciones físicas y/o materiales (compuesta por los ítems 1, 13, 21, 28 y 32).
2. Beneficios laborales y/o remunerativos (compuesta por los ítems 2, 7, 14 y 22).

3. Políticas administrativas (compuesta por los ítems 8, 15, 17,23 y 33).

4. Relaciones sociales (compuesta por los ítems 3, 9, 16 y 24).

5. Desarrollo personal (compuesta por los ítems 4, 10, 18, 25, 29 y 34).

6. Desempeño de tareas (compuesta por los ítems 5, 11, 19, 26, 30 y 35 .

7. Relación con la autoridad (compuesta por los ítems 6, 12, 20, 27, 31 y 36 ).

La validez de constructo se obtuvo mediante análisis factorial exploratorio y su índice de confiabilidad supera el .7 para toda la prueba, el cual fue obtenido mediante el método de consistencia interna con la prueba Alfa de Cronbach. Para valorar los niveles de satisfacción laboral, se consideraron las categorías diagnósticas dadas por Palma (1999), como se tiene en la Tabla 1. 
Tabla 1. Categorías diagnósticas de la SL-SPC

\begin{tabular}{|c|c|c|c|c|c|c|c|c|}
\hline $\begin{array}{l}\text { Satisfacción } \\
\text { laboral }\end{array}$ & $F_{1}$ & $F_{2}$ & $F_{3}$ & $F_{4}$ & $\mathrm{~F}_{5}$ & F6 & $\mathrm{F}_{7}$ & P.T. \\
\hline $\begin{array}{l}\text { Alta } \\
\text { satisfacción }\end{array}$ & $23 a+$ & $18 \mathrm{a}+$ & $23 a+$ & $19 a+$ & $29 a+$ & $29 a+$ & $29 a+$ & $168 a+$ \\
\hline $\begin{array}{l}\text { Parcial } \\
\text { satisfacción } \\
\text { laboral }\end{array}$ & 20 a 22 & 15 a 17 & 20 a 22 & 17 a 18 & 26 a 28 & 26 a 28 & 25 a 28 & 149 a 167 \\
\hline Regular & 15 a 19 & 9 a 14 & 15 a 19 & 12 a 16 & 19 a 25 & 20 a 25 & 19 a 24 & 112 a 148 \\
\hline $\begin{array}{l}\text { Parcial } \\
\text { insatisfacción } \\
\text { laboral }\end{array}$ & 11 a 14 & 7 a 8 & 11 a 14 & 8 a 11 & 14 a 18 & 14 a 19 & 14 a 18 & 93 a 111 \\
\hline $\begin{array}{l}\text { Alta } \\
\text { insatisfacción }\end{array}$ & $10 \mathrm{a}-$ & $6 a-$ & $7 a-$ & $13 a-$ & $13 a-$ & $13 a-$ & $13 a-$ & $92 \mathrm{a}-$ \\
\hline
\end{tabular}

\section{Procedimiento}

Los trabajadores fueron evaluados en un centro de salud ocupacional, como parte de las evaluaciones rutinarias que exige realizar una vez al año, el Estado peruano. Los cuestionarios se estructuraron con una hoja de evaluación que comprende los datos sociodemográficos, como son edad, área de trabajo y cargo. Todos los participantes fueron informados sobre los fines del estudio y estuvieron dispuestos a colaborar con él, ellos firmaron un consentimiento informado y decidieron participar de forma voluntaria, siempre que sus datos personales no fueran revelados. Las evaluaciones se aplicaron de forma individual a cada participante en el turno de la mañana. Una vez recogidos los datos fueron procesados estadísticamente.

\section{Análisis de Datos}

Para el análisis y procesamiento se utilizó el programa SPSS versión 23. Se utilizaron pruebas estadísticas tales como el análisis de varianza para las comparaciones según los criterios de comparación, que fueron la edad, el cargo y el área de trabajo.

\section{Resultados}

En la tabla 2 se puede apreciar que la dimensión Condiciones físicas de la satisfacción laboral tiene una media de 18.36 con una desviación estándar de 4.05, dentro de un rango de 5a 25. La dimensión de Beneficios laborales obtuvo una media de 12.59 con una desviación estándar de 2.95 dentro de un rango de 5 a 20 . La dimensión de Políticas administrativas obtuvo una media de 18.14 con una desviación estándar de 3.74 dentro de un rango de 9 a 25.

La dimensión de Relaciones sociales tiene una media de 17.02 con una desviación estándar de 2.14 dentro de un rango de 9 a 20. La dimensión Desarrollo personal tiene una media de 25.60 con una desviación estándar de 2.68 dentro de un rango de 20 a 30. La dimensión Desempeño de tareas tiene una media de 26.51 con una desviación estándar de 2.46 dentro de un 
rango de 20 a 30. La dimensión Relación con la autoridad tiene una media de 22.12 con una desviación estándar de 4.83 dentro de un rango de 6 a 30. La dimensión Satisfacción laboral obtuvo una media de 140.32 con una desviación estándar de 16.06 dentro de un rango de 100 a 180 . Las asimetrías y las curtosis de todas las dimensiones tienen valores inferiores a 1.5, lo que supone una tendencia hacia la distribución normal de los datos.

Tabla 2. Estadísticos descriptivos

\begin{tabular}{|c|c|c|c|c|c|c|c|c|}
\hline & 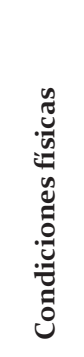 & 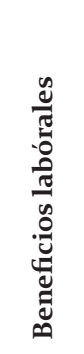 & 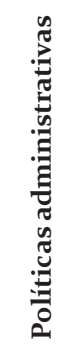 & 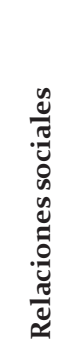 & 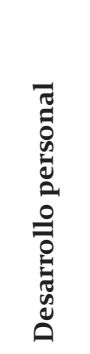 & 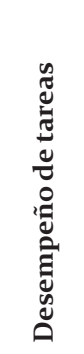 & 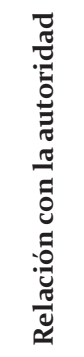 & 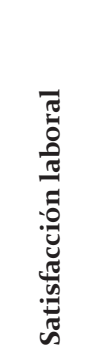 \\
\hline Media & 18.36 & 12.59 & 18.14 & 17.02 & 25.60 & 26.51 & 22.12 & 140.32 \\
\hline Mediana & 19.00 & 13.00 & 19.00 & 17.00 & 25.00 & 26.00 & 23.00 & 141.00 \\
\hline Moda & 20.00 & 14.00 & 20.00 & 17.00 & 24.00 & 25.00 & 24.00 & 143.00 \\
\hline DE & 4.05 & 2.95 & 3.74 & 2.14 & 2.68 & 2.46 & 4.83 & 16.06 \\
\hline Varianza & 16.42 & 8.70 & 14.05 & 4.58 & 7.21 & 6.05 & 23.37 & 258.10 \\
\hline Asimetría & -0.96 & 0.01 & -0.38 & -1.17 & 0.02 & -0.23 & -0.89 & 0.17 \\
\hline Curtosis & 1.25 & 0.54 & 0.02 & 2.73 & -0.66 & -0.53 & 1.02 & 0.24 \\
\hline Rango & 20.00 & 15.00 & 16.00 & 11.00 & 10.00 & 10.00 & 24.00 & 80.00 \\
\hline Mínimo & 5.00 & 5.00 & 9.00 & 9.00 & 20.00 & 20.00 & 6.00 & 100.00 \\
\hline Máximo & 25.00 & 20.00 & 25.00 & 20.00 & 30.00 & 30.00 & 30.00 & 180.00 \\
\hline
\end{tabular}

En la Figura 1. En cuanto a los niveles de satisfacción laboral, se tiene que en la dimensión de Condiciones físicas el 14.5\% tienealta satisfacción, el 12\% poca insatisfacción, el 36.1\% tieneuna satisfacción regular, el 32.5\% tiene poca satisfacción laboral y el $4.8 \%$ baja satisfacción. En la dimensión Beneficios laborales el $4.8 \%$ tiene alta satisfacción, el 8.4\% tiene poca insatisfacción, el 71.1\% tiene una satisfacción regular, el 13.3\% tiene poca satisfacción y el 2.4\% tiene baja satisfacción. En la dimensión Políticas administrativas el 9.6\% tiene alta satisfacción, el 13.3\% tiene poca insatisfacción, el $42.2 \%$ tiene una satisfacción regular, el $30.1 \%$ tiene poca satisfacción y el $4.8 \%$ tiene baja satisfacción.

En la dimensión Relaciones sociales el $22.9 \%$ tiene alta satisfacción, el $2.4 \%$ tiene poca satisfacción, el 32,5\% tiene regular satisfacción, el 42.2\% tiene poca satisfacción. En la dimensión desarrollo personal el 18,1\% tiene alta satisfacción, el 
50.6\%tiene una satisfacción regular y el 31.3\% tiene poca satisfacción. En la dimensión desempeño de tareas el $26.5 \%$ tiene alta satisfacción, el $38.6 \%$ tiene una satisfacción regular y el $34.9 \%$ tiene poca satisfacción. En la dimensión relación con la autoridad el $6 \%$ tiene alta satisfacción, el
$12 \%$ tiene poca insatisfacción, el 53\% tiene una satisfacción regular, el 22.9\% tiene poca satisfacción y el $6 \%$ tiene baja satisfacción. En la dimensión Satisfacción laboral el 6\% tiene alta satisfacción, el 3.6\% tiene poca insatisfacción, el $71.1 \%$ regular satisfacción y el $19.3 \%$ poca satisfacción.

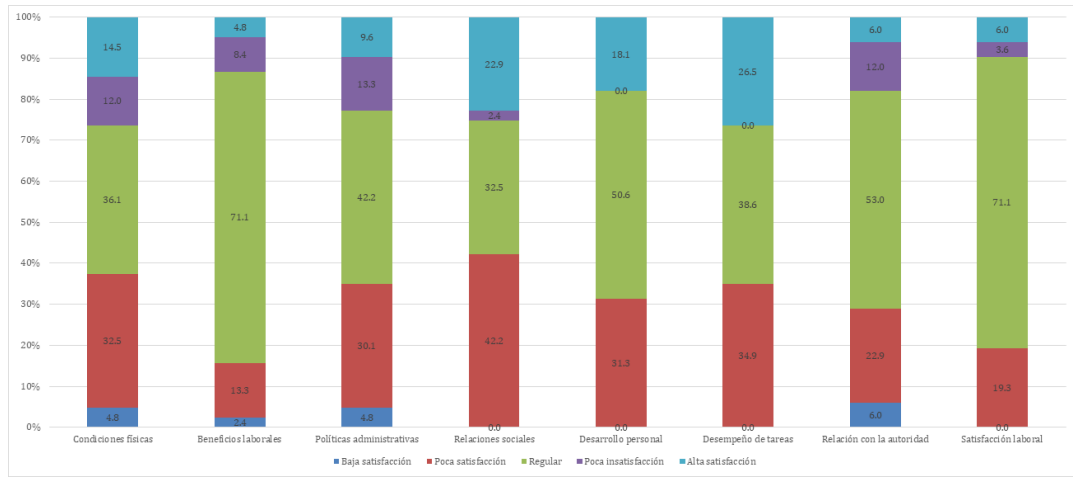

Figura 1. Niveles de satisfacción laboral por dimensiones

En la Tabla 3, se tienen las comparaciones de la satisfacción laboral y sus dimensiones en función de la edad de los trabajadores. En ese sentido, se formaron tres categorías de edad para realizar las comparaciones. En la primera categoría, se tienen los trabajadores que tienen entre 24 y 30 años y que constituyen el $32.5 \%$ de la muestra $(n=27)$, en la categoría de entre 31 y 40 años se tiene el $44.6 \%$ de la muestra $(n=37)$ y en la categoría de entre 41 y 62 años se tiene el $22.9 \%$ de la muestra $(n=19)$. Las comparaciones efectuadas con la prueba de análisis de varianza revelan que no existen diferencias significativas $(\mathrm{p}<0.05)$ en ninguna de las dimensiones de la satisfacción laboral, ni en el puntaje global según la edad. 
Tabla 3. Análisis de varianza según la edad

\begin{tabular}{|c|c|c|c|c|c|c|}
\hline & & $\begin{array}{c}\text { Sumade } \\
\text { cuadrados }\end{array}$ & gl & $\begin{array}{c}\text { Media } \\
\text { cuadrática }\end{array}$ & $\mathbf{F}$ & Sig. \\
\hline \multirow{3}{*}{ Condiciones físicas } & Entre grupos & 51.387 & 2 & 25.694 & 1.586 & 0.211 \\
\hline & Dentro de grupos & $1295 \cdot 770$ & 80 & 16.197 & & \\
\hline & Total & 1347.157 & 82 & & & \\
\hline \multirow{3}{*}{ Beneficios laborales } & Entre grupos & 26.282 & 2 & 13.141 & 1.529 & 0.223 \\
\hline & Dentro de grupos & 687.790 & 80 & 8.597 & & \\
\hline & Total & 714.072 & 82 & & & \\
\hline \multirow{3}{*}{$\begin{array}{l}\text { Políticas } \\
\text { administrativas }\end{array}$} & Entre grupos & 22.169 & 2 & 11.084 & 0.785 & 0.460 \\
\hline & Dentro de grupos & 1130.097 & 80 & 14.126 & & \\
\hline & Total & 1152.265 & 82 & & & \\
\hline \multirow{3}{*}{ Relaciones sociales } & Entre grupos & 7.134 & 2 & 3.567 & 0.774 & 0.465 \\
\hline & Dentro de grupos & 368.817 & 80 & 4.610 & & \\
\hline & Total & 375.952 & 82 & & & \\
\hline \multirow{3}{*}{ Desarrollo personal } & Entre grupos & 14.316 & 2 & 7.158 & 0.991 & 0.376 \\
\hline & Dentro de grupos & 577.563 & 80 & 7.220 & & \\
\hline & Total & 591.880 & 82 & & & \\
\hline \multirow{3}{*}{ Desempeño tareas } & Entre grupos & 8.609 & 2 & 4.305 & 0.705 & 0.497 \\
\hline & Dentro de grupos & 488.114 & 80 & 6.101 & & \\
\hline & Total & 496.723 & 82 & & & \\
\hline \multirow{3}{*}{ Relación autoridad } & Entre grupos & 111.493 & 2 & $55 \cdot 746$ & 2.470 & 0.091 \\
\hline & Dentro de grupos & $1805 \cdot 302$ & 80 & 22.566 & & \\
\hline & Total & 1916.795 & 82 & & & \\
\hline \multirow{3}{*}{ Satisfacción laboral } & Entre grupos & 766.701 & 2 & 383.351 & 1.504 & 0.229 \\
\hline & Dentro de grupos & 20397.516 & 80 & 254.969 & & \\
\hline & Total & 21164.217 & 82 & & & \\
\hline
\end{tabular}

En la Tabla 4 se tienen las comparaciones según el cargo de los trabajadores, para lo cual se empleó el análisis de varianza, y se reportaron diferencias estadísticamente significativas en varias dimensiones de la satisfacción laboral, tales como Condiciones físicas $(\mathrm{p}=0.006)$, Beneficios laborales $(\mathrm{p}=0.005)$, políticas administrativas $(\mathrm{p}=0.041)$, relaciones sociales $(\mathrm{p}=\mathrm{0.044})$, desarrollo personal $(\mathrm{p}=0.040)$, relación con la autoridad $(\mathrm{p}=$ o.0o9) y la satisfacción laboral como puntuación total $(\mathrm{p}=\mathrm{o.001})$; de modo que los trabajadores que tienen cargos de menor jerarquía como los ayudantes de grúa, ayudantes mecánicos, asistentes de patio, operadores de tapas, operadores de remolcador, maestros electricistas y maestros mecánicos, tienen puntajes inferiores en las dimensiones señaladas 
en comparación con los de mayor jerar- jefes de seguridad integral, los coordiquía que son jefes de patio, jefes de alma- nadores y los administradores de base cén, jefes de tren, jefes de transportes, de datos.

Tabla 4. Análisis de varianza según el cargo

\begin{tabular}{|c|c|c|c|c|c|c|}
\hline & & $\begin{array}{c}\text { Suma de } \\
\text { cuadrados }\end{array}$ & gl & $\begin{array}{c}\text { Media } \\
\text { cuadrática }\end{array}$ & F & Sig. \\
\hline \multirow{3}{*}{$\begin{array}{l}\text { Condiciones } \\
\text { físicas }\end{array}$} & Entre grupos & $595 \cdot 301$ & 21 & 28.348 & 2.300 & 0.006 \\
\hline & Dentro de grupos & 751.856 & 61 & 12.326 & & \\
\hline & Total & 1347.157 & 82 & & & \\
\hline \multirow{3}{*}{$\begin{array}{l}\text { Beneficios } \\
\text { laborales }\end{array}$} & Entre grupos & 319.043 & 21 & 15.193 & 2.346 & 0.005 \\
\hline & Dentro de grupos & 395.029 & 61 & 6.476 & & \\
\hline & Total & 714.072 & 82 & & & \\
\hline \multirow{3}{*}{$\begin{array}{l}\text { Políticas } \\
\text { administrativas }\end{array}$} & Entre grupos & 438.717 & 21 & 20.891 & 1.786 & 0.041 \\
\hline & Dentro de grupos & 713.548 & 61 & 11.698 & & \\
\hline & Total & 1152.265 & 82 & & & \\
\hline \multirow{3}{*}{$\begin{array}{l}\text { Relaciones } \\
\text { sociales }\end{array}$} & Entre grupos & 142.168 & 21 & 6.770 & 1.766 & 0.044 \\
\hline & Dentro de grupos & 233.783 & 61 & 3.833 & & \\
\hline & Total & $375 \cdot 95^{2}$ & 82 & & & \\
\hline \multirow{3}{*}{$\begin{array}{l}\text { Desarrollo } \\
\text { personal }\end{array}$} & Entre grupos & 225.962 & 21 & 10.760 & 1.794 & 0.040 \\
\hline & Dentro de grupos & 365.917 & 61 & 5.999 & & \\
\hline & Total & 591.880 & 82 & & & \\
\hline \multirow{3}{*}{$\begin{array}{l}\text { Desempeño } \\
\text { tareas }\end{array}$} & Entre grupos & 146.010 & 21 & 6.953 & 1.209 & 0.276 \\
\hline & Dentro de grupos & 350.713 & 61 & 5.749 & & \\
\hline & Total & 496.723 & 82 & & & \\
\hline \multirow{3}{*}{$\begin{array}{l}\text { Relación } \\
\text { autoridad }\end{array}$} & Entre grupos & 825.799 & 21 & $39 \cdot 324$ & 2.199 & 0.009 \\
\hline & Dentro de grupos & 1090.996 & 61 & 17.885 & & \\
\hline & Total & 1916.795 & 82 & & & \\
\hline \multirow{3}{*}{$\begin{array}{l}\text { Satisfacción } \\
\text { laboral }\end{array}$} & Entre grupos & 10348.555 & 21 & 492.788 & 2.779 & 0.001 \\
\hline & Dentro de grupos & 10815.662 & 61 & $177 \cdot 306$ & & \\
\hline & Total & 21164.217 & 82 & & & \\
\hline
\end{tabular}

En la Tabla 5 podemos apreciar las comparaciones en función del área de trabajo de los trabajadores mediante el análisis de varianza. De este modo, en la dimensión Condiciones físicas de trabajo, se registraron diferencias significativas $(\mathrm{p}=0.000)$ siendo que los trabajadores del área de mecánica tienen una media de 19.93, que los del área administrativa 17.25 y que los trabajadores del área de transporte 16.18. En la dimensión Beneficios Laborales se registraron diferencias significativas ( $\mathrm{p}=0.007$ ) siendo que los del área administrativa tienen una media de 15.5, los 
del área de Mecánica 13.08 y los trabaja- o.0oo) siendo los del área administrativa, dores del área de transportes 11.5. En la quienes tienen una media de 21.0, los del dimensión políticas administrativas se área de mecánica 19.29y los trabajadores registraron diferencias significativas ( $\mathrm{p}=$ del área de transporte 16.09.

Tabla 5. Análisis de varianza en función del área de trabajo

\begin{tabular}{|c|c|c|c|c|c|c|}
\hline & Cargo & $\mathbf{N}$ & Media & gl & $\mathbf{F}$ & Sig. \\
\hline \multirow{3}{*}{$\begin{array}{l}\text { Condiciones } \\
\text { físicas }\end{array}$} & Administrativos & 4 & 17.25 & 2 & 10.153 & 0.000 \\
\hline & Transporte & 32 & 16.18 & 80 & & \\
\hline & Mecánicos & 47 & 19.93 & 82 & & \\
\hline \multirow{3}{*}{$\begin{array}{l}\text { Beneficios } \\
\text { laborales }\end{array}$} & Administrativos & 4 & $15 \cdot 5$ & 2 & 5.291 & 0.007 \\
\hline & Transporte & 32 & 11.5 & 80 & & \\
\hline & Mecánicos & 47 & 13.08 & 82 & & \\
\hline \multirow{3}{*}{$\begin{array}{l}\text { Políticas } \\
\text { administrativas }\end{array}$} & Administrativos & 4 & 21.0 & 2 & 9.960 & 0.000 \\
\hline & Transporte & 32 & 16.09 & 80 & & \\
\hline & Mecánicos & 47 & 19.29 & 82 & & \\
\hline \multirow{3}{*}{$\begin{array}{l}\text { Relaciones } \\
\text { sociales }\end{array}$} & Administrativos & 4 & 18.5 & 2 & 2.436 & 0.094 \\
\hline & Transporte & 32 & 16.46 & 80 & & \\
\hline & Mecánicos & 47 & 17.27 & 82 & & \\
\hline \multirow{3}{*}{$\begin{array}{l}\text { Desarrollo } \\
\text { personal }\end{array}$} & Administrativos & 4 & 27.75 & 2 & 3.063 & 0.052 \\
\hline & Transporte & 32 & 24.84 & 80 & & \\
\hline & Mecánicos & 47 & 25.93 & 82 & & \\
\hline \multirow{3}{*}{$\begin{array}{l}\text { Desempeño } \\
\text { de tareas }\end{array}$} & Administrativos & 4 & 28.5 & 2 & 1.376 & 0.259 \\
\hline & Transporte & 32 & 26.4 & 80 & & \\
\hline & Mecánicos & 47 & 26.42 & 82 & & \\
\hline \multirow{3}{*}{$\begin{array}{l}\text { Relación con la } \\
\text { autoridad }\end{array}$} & Administrativos & 4 & 26 & 2 & $17 \cdot 527$ & 0.000 \\
\hline & Transporte & 32 & 18.81 & 80 & & \\
\hline & Mecánicos & 47 & 24.02 & 82 & & \\
\hline \multirow{3}{*}{$\begin{array}{l}\text { Satisfacción } \\
\text { laboral }\end{array}$} & Administrativos & 4 & $154 \cdot 5$ & 2 & 14.789 & 0.000 \\
\hline & Transporte & 32 & 130.09 & 80 & & \\
\hline & Mecánicos & 47 & 146.08 & 82 & & \\
\hline
\end{tabular}

En la dimensión Relaciones Sociales, área administrativa tienen una media de Desarrollo personal y Desempeño de 26, los delárea mecánica 24.02 y los trabatareas, no se registraron diferencias signi- jadores del área de transporte 18.81. En la ficativas. En la dimensión Relación con dimensión Satisfacción laboral se registrala autoridad se registraron diferencias ron diferencias significativas $(p=0.000)$ significativas $(\mathrm{p}=0.000)$ siendo que los del siendo que los del área administrativa 
tienen una media de 154.5, los del área de mecánica 146.08 y los trabajadores del área de transporte 130.09.

\section{Discusión}

La presente investigación está centrada en encontrar diferencias en el nivel de satisfacción laboral en cuanto a variables sociodemográficas tales como edad, área de trabajo y cargo en los trabajadores de una empresa ferroviaria. Como se indicó previamente, no se encontraron diferencias significativas en cuanto a la edad, lo cual contradice algunos estudios que señalan que son los trabajadores de menor edad (Argyle, 1987; Fernández-Ballesteros, 1997), o de mayor edad (Robina, 2002; Alonso, 2006) los que se sienten más satisfechos.

Por otro lado, de acuerdo con los datos obtenidos en la investigación se puede concluir con respecto a los niveles de satisfacción laboral, según los factores del modelo de Palma (1999), 36.1\% presenta una satisfacción regular en Condiciones físicas, lo cual significa que un alto porcentaje de los trabajadores se encuentra regularmente satisfecho con los elementos ya sean materiales o de infraestructura, en los que se desenvuelven diariamente. En la dimensión Beneficios laborales, encontramos que el $71.1 \%$ tiene una satisfacción laboral regular, y en la dimensión Políticas administrativas, el $42.2 \%$ tiene una satisfacción laboral regular, lo cual significa que los trabajadores se encuentran regularmente satisfechos ante las reglas o normas que rigen la institución. En cuanto a la dimensión Relaciones sociales se encuentra que el $42.2 \%$ tiene poca satisfacción, lo cual refleja que los trabajadores se encuentran poco satisfechos con respecto al trato que se tiene con sus compañeros de trabajo dentro de la organización. En la dimensión Desarrollo personal, que se relaciona con las oportunidades que tienen dentro de la empresa para poder autorrealizarse, el 50.6\% tiene una satisfacción laboral regular, mientras que la dimensión Desempeño de tareas el $38.6 \%$ tiene una satisfacción regular. En la dimensión Relación con la autoridad el $53 \%$ tiene una satisfacción regular lo cual significa que los trabajadores se encuentran regularmente satisfechos con el valor que le dan a la relación con sus superiores.

En conclusión, puede decirse que los trabajadores evaluados se encuentran moderadamente satisfechos en la mayoría de factores evaluados, lo que es consistente con el que el $71.1 \%$ de los trabajadores se ubica en un nivel regular de satisfacción laboral. Pero en cuento al cargo y el área de trabajo, se encontró que en la dimensión Condiciones físicas, los que trabajan en el área mecánica presentan una mayor satisfacción a comparación de las otras áreas. Por otro lado, en las dimensiones Beneficios laborales, Políticas administrativas y Relación con la autoridad, se registró que son los trabajadores del área administrativa quienes muestran un mayor nivel de satisfacción laboral, respecto a las otras áreas. Se concluye, por tanto, que en cuanto al cargo, los trabajadores del área administrativa se encuentran más satisfechos que los trabajadores del área mecánica y trabajadores del área de transporte. Esto podría explicarse, debido 
a que los trabajadores administrativos están menos expuestos a riesgos de seguridad en comparación con los trabajadores del área mecánica y ferroviaria (Probst, 2000), pero también, porque el área administrativa comprende a los jefes de área que por la jerarquía de su cargo podrían tener mejores beneficios laborales, salarios más altos y mayor comodidad para la realización de sus funciones; aspectos asociados positivamente a la satisfacción laboral (Peiró, 1984).

Este estudio es relevante porque puede ser el punto de partida para futuras investigaciones sobre el tema de la satisfacción laboral y su asociación con diversas variables sociodemográficas; pero se sugiere que se puedan tomar muestras representativas de trabajadores de diferentes empresas y rubros laborales, así como diversas variables sociodemográficas para tomarlas como criterio de comparación, tales como el estado civil, el salario, el grado de instrucción, etc. En ese sentido, una de las limitaciones en este estudio, se refiere a que no se han tomado estas medidas ni se ha seguido un muestreo probabilístico, por tanto, los resultados hallados solo se circunscriben a la empresa de donde proviene la muestra; pero sería conveniente profundizar en esta temática en nuestra localidad, ya que aunque se habla mucho de la satisfacción laboral, son pocos los estudios a nivel nacional sobre esta variable, tan importante en el ámbito organizacional. 


\section{Referencias}

Adams, J. S. (1963). Towards an understanding of inequity. The Journal of Abnormal and Social Psychology, 67(5), 422-436.

Alderfer, C. P. (1969). An empirical test of a new theory of human needs. Organizational Behavior E Human Performance, 4(2), 142-175.

Alonso, P. (2006). Diferencias en la percepción de la satisfacción laboral en una muestra de personal de administración. Boletín de Psicología, 88, 49-64.

Alonso, P. (2008). Estudio comparativo de la satisfacción laboral en el personal de administración. Revista de Psicología del Trabajo y de las Organizaciones, 24(1), 25-40.

Argyle, M. (1987). The psychology of happiness. New York: Methuen.

Arias, W. L, \& Justo. O. (2013). Satisfacción laboral en trabajadores de dos tiendas por departamento. Un estudio comparativo. Ciencia $\mathcal{E}$ Trabajo, 15(47), 41-46.

Ato, M., López, J. J., \& Benavente, A. (2013). Un sistema de clasificación de los diseños de investigación en psicología. Anales de Psicología, 29(3), 1038-1059.

Borjas, G. J. (1979). Job Satisfaction, wages and Unions. Journal of Human Resources, 14, 21-40.

Brugemann, A. (1974). Distinción entre distintas formas de satisfacción. Trabajo y Rendimiento, 28, 281-284.

Carrillo-García, C., Martínez-Roche, M. E., Gómez-García, C. I., \& Meseguer-dePedro, M. (2015). Satisfacción laboral de los profesionales sanitarios de un Hospital Universitario: análisis general y categorías laborales. Anales de Psicología, 31(2), 645-650.

Chiavenato, I. (2009). Gestión del talento humano. México: McGraw-Hill.

Clemente, A., Molero, R., \& González, F. (2000). Estudio de la satisfacción personal según la edad de las personas. Anales de Psicología, 16(2), 189-198.

Fairbrother, K., \& Warn, J. (2003). Workplace dimensions, stress and job satisfaction. Journal of Managerial Psychology, 18(1), 8-21. 
Fernández-Ballesteros, R. (1997). Calidad de vida en la vejez: Condiciones diferenciales. Intervención Psicosocial, 6(1), 21-35.

Gamero, H. (2013). La satisfacción laboral como dimensión de la felicidad. Ciencia E Trabajo, 15(47), 94-102.

Hackman, J. R., \& Oldham, G. R (1976). Motivation through the design of work: Test of a theory. Organizational Behavior and Human Performance, 16, 250-279.

Hong, S. M., \& Giannakopulos, E. (1994). Effects of age, sex and university status on life-satisfaction. Psychological Reports, 74(1), 99-103.

Hoppock, R. (1935). Job Satisfaction. New York: Harper.

Herzberg, F. (2004). Una vez más: ¿Cómo se motiva a los empleados? En Harvard Business Review (pp. 53-80). Barcelona: Deusto.

Herzberg, F., Mausner, B., \& Snyderman, B. (1959). The Motivation to Work. New York: John Wiley \& Sons, Inc.

León, F. (1981). The role of positive and negative outcomes in the causation of motivational forces. Journal of Applied Psychology, 66, 45-53.

León, F., \& Sepúlveda, M. V. (1979). Satisfacciones e insatisfacciones herzbergianas en el trabajo. Revista Latinoamericana de Psicología, 11(1), 93-113.

Locke, E. (1968). What is job satisfactory? Documento de trabajo presentado en la American Psycological Association Convention, San Francisco, California.

Locke, E. A. (1976). The nature and causes of jobsatisfaction. En M. D. Dunnette (Ed.) Handbook of Industrial and Organizational Psychology. Chicago: Rand McNally.

Locke, E. A. (1984). Job satisfaction. En M. Gruneberg y T. Wall (Eds.) Social Psychology and Organizational Behaviour. Chichester: Wiley.

Maslow, A. H. (1943). A theory of human motivation. Psychological Review, 50(4), 370-396. doi: 10.1037/hoo54346

Palma, S. (1999). Elaboración y validación de una escala de satisfacción laboral SL-SPC para trabajadores de Lima Metropolitana. Teoría e Investigación en Psicología, 9(1), 27-34. 
Peiró, J. M. (1984). Psicología de la organización. Madrid: UNED.

Pérez-Ciordia, I., Guillén-Grima, F., Brugos, A., \& Aguinaga, I. (2013). Satisfacción laboral y factores de mejora en profesionales de atención primaria. Anuario de Sistema Sanitario de Navarra, 36, 253-262.

Probst, T. M. (200o). Wedded to the job: Moderating effects of job involvement on the consequences of job insecurity. Journal of Occupational Health Psychology, $5,63-73$.

Robina, R. (2002). Condiciones sociolaborales de los empleados públicos: Motivación y satisfacción laboral en la administración regional de Extremadura. (Tesis doctoral). Universidad de Extremadura, España.

Rodríguez, P. (2009). Índice de satisfacción laboral de los empleados contingentes en una cadena de restaurantes de comida rápida en el área suroeste de Puerto Rico. (Tesis de Maestría). Universidad de Puerto Rico, Mayagüez, Puerto Rico.

Schultz, D. (1991). Psicología industrial. México: Prentice Hall.

Sousa, V. D., Driessnack, M., \& Mendes, I. A. C. (2007). Revisión de diseños de investigación resaltantes para enfermería. Parte 1: Diseños de investigación cuantitativa. Revista Latino-Americana de Enfermagen, 15(3), 502-507.

Recibido: 26 de setiembre de 2019

Aceptado: 2 de junio de 2020 